\title{
Somatic mosaicism detected by exon-targeted, high-resolution aCGH in 10362 consecutive cases
}

\author{
Justin Pham ${ }^{1}$, Chad Shaw ${ }^{1}$, Amber Pursley ${ }^{1}$, Patricia Hixson ${ }^{1}$, Srirangan Sampath ${ }^{1}$, Erin Roney ${ }^{1}$, \\ Tomasz Gambin ${ }^{1}$, Sung-Hae L Kang ${ }^{2}$, Weimin Bi $^{1}$, Seema Lalani ${ }^{1,3}$, Carlos Bacino ${ }^{1,3}$, James R Lupski ${ }^{1,3,4}$, \\ Pawel Stankiewicz ${ }^{1}$, Ankita Patel $^{1}$ and Sau-Wai Cheung ${ }^{\star, 1}$
}

Somatic chromosomal mosaicism arising from post-zygotic errors is known to cause several well-defined genetic syndromes as well as contribute to phenotypic variation in diseases. However, somatic mosaicism is often under-diagnosed due to challenges in detection. We evaluated 10362 patients with a custom-designed, exon-targeted whole-genome oligonucleotide array and detected somatic mosaicism in a total of 57 cases $(0.55 \%)$. The mosaicism was characterized and confirmed by fluorescence in situ hybridization (FISH) and/or chromosome analysis. Different categories of abnormal cell lines were detected:

(1) aneuploidy, including sex chromosome abnormalities and isochromosomes (22 cases), (2) ring or marker chromosomes (12 cases), (3) single deletion/duplication copy number variations (CNVs) (11 cases), (4) multiple deletion/duplication CNVs (5 cases), (5) exonic CNVs (4 cases), and (6) unbalanced translocations (3 cases). Levels of mosaicism calculated based on the array data were in good concordance with those observed by FISH (10-93\%). Of the 14 cases evaluated concurrently by chromosome analysis, mosaicism was detected solely by the array in 4 cases (29\%). In summary, our exon-targeted array further expands the diagnostic capability of high-resolution array comparative genomic hybridization in detecting mosaicism for cytogenetic abnormalities as well as small CNVs in disease-causing genes.

European Journal of Human Genetics (2014) 22, 969-978; doi:10.1038/ejhg.2013.285; published online 8 January 2014

Keywords: somatic mosaicism; array CGH; CNV; chromosomal structural abnormalities; mosaic exon deletion

\section{INTRODUCTION}

Somatic chromosomal mosaicism is defined as the presence of two or more chromosomally distinct cell lines and represents a challenging task not only to detect but also to interpret. Owing to various factors including subtle phenotypic abnormalities, technical limitations, and inherent tissue-specificity, mosaicism is often undetected and underdiagnosed.

Advances in chromosomal microarray analysis (CMA) have transformed the microarray into a powerful genome-wide analysis tool able to detect a wide range of aberrations of the human genome in a clinical setting. Detection of mosaicism by CMA for aneuploidy as well as structural abnormalities has been previously reported. ${ }^{1-4}$ In 2006 and 2007, Ballif et al. and Cheung et al. each concluded in their respective retrospective studies that the use of CMA via array comparative genomic hybridization (CGH) is more sensitive than traditional cytogenetics in the likelihood of detecting mosaicism and provides a more accurate first tier test. ${ }^{1,2}$ However, Bi et al. recently presented evidence for the value of chromosome studies in conjunction with CMA to maximize the detection of mosaicism, citing the shortcomings of CMA in detecting low levels of mosaicism $(<10 \%)$ for either abnormal or normal cell lines, and the presence of two or more abnormal cell lines involving the same region. ${ }^{5}$ In their study, six $(0.16 \%)$ cases of mosaic abnormalities identified by chromosome analysis were undetected by CMA, four of which had a level of mosaicism $<10 \%$ supporting the concern that array technologies are not reliable for routinely detecting very low-level mosaicism. However, mosaicism for whole chromosome aneuploidy, the most common type of mosaicism, ${ }^{6}$ has been detected as low as $5 \%$ by both $\mathrm{CMA}^{2}$ and single nucleotide polymorphism (SNP) arrays. ${ }^{3}$ In 2007, $\mathrm{Lu}$ et al. reported detection of a case of low level mosaicism for trisomy 14 by a bacterial artificial chromosome (BAC) array that subsequently was validated by fluorescence in situ hybridization (FISH) on cultured cells and chromosome analysis finding a mosaicism level of $2 \%{ }^{7}$

Until recently, most clinical array platforms have used either oligonucleotides or SNPs as probes, each having an advantage over the other with regard to detecting specific types of abnormalities that can be present in the mosaic state. Specifically, SNP arrays provide data not attainable by oligonucleotide-based CMA arrays such as loss of heterozygosity and copy-number-neutral changes which allows for detection of uniparental disomy. CMA has the flexibility of routinely detecting very small mosaic copy number changes such as intragenic, exonic copy number variants (CNVs), which can be limited in SNP arrays due to the random distribution of SNPs across the genome. ${ }^{5,8}$ Therefore, to maximize detection, oligonucleotide platforms that include SNPs are becoming the prevalent array used for clinical diagnostics. ${ }^{3,4,9,10}$

${ }^{1}$ Department of Molecular and Human Genetics, Baylor College of Medicine, Houston, TX, USA; ${ }^{2}$ Allina Medical Laboratories, Minneapolis, MN, USA; ${ }^{3}$ Texas Children's Hospital, Houston, TX, USA; ${ }^{4}$ Department of Pediatrics, Baylor College of Medicine, Houston, TX, USA

*Correspondence: Professor S-W Cheung, Department of Molecular and Human Genetics, Baylor College of Medicine-Medical Genetics Laboratories, One Baylor Plaza, Houston, TX 77030, USA. Tel: +1 713798 4991; Fax: + 1713798 2787; E-mail: scheung@bcm.edu

Received 12 April 2013; revised 8 November 2013; accepted 20 November 2013; published online 8 January 2014 
In 2009, our clinical diagnostic molecular cytogenetics laboratory added exonic coverage for over 1700 known disease and candidate genes to our custom-designed oligonucleotide array, making it possible to detect mosaicism for a CNV within a single gene. Four cases of mosaicism involving a $\mathrm{CNV}$ within a single gene were identified using this array and included in this study, two of which have previously been reported. ${ }^{8}$ Recently, there have been several reports of exonic mosaicism detected by array $\mathrm{CGH}^{11-13}$ underscoring the importance of detecting this type of aberration. For example, Castronovo et al. described a patient who presented with an atypical phenotype and, following array CGH, a low-level mosaic intragenic NSD1 deletion was discovered ultimately resulting in a diagnosis of Sotos syndrome. ${ }^{11}$ Saitsu et al. described a patient with a microdeletion involving a portion of the CASK gene that was inherited from his apparently healthy mother who had somatic mosaicism (20\%) for the deletion. ${ }^{12}$ Bartnik et al. report the first cases (three cases) of mosaic exonic deletions of the gene CDKL5 whose mutations have been associated with X-linked dominant early infantile epileptic encephalopathy- $2 .{ }^{13}$

Here we present the cases of mosaicism detected in a clinical cohort of 10362 consecutive patients referred for clinical testing using our custom-designed, exon-targeted whole-genome oligonucleotide array. Specifically, our study highlights those cases with complex genomic rearrangements, multiple abnormal cells lines, exonic CNVs and where conventional cytogenetic and CMA methodologies are discordant.

\section{MATERIALS AND METHODS}

\section{Subjects}

In total, 10362 consecutive patient samples were submitted to the Medical Genetics Laboratories at Baylor College of Medicine for CMA analysis during the period from June 2009 to February 2011. In all, 1063 of these cases that had concurrent CMA and chromosome analysis have been previously reported by Bi et al..$^{5}$ The indication for ordering CMA was provided for 8898 cases. In total, 5138 patients studied had a clinical indication of cognitive impairment (primarily developmental delay, intellectual disability, and autism). This study focuses on the 57 cases in which mosaicism was detected by CMA using the version 8 oligoarray (V8 OLIGO).

\section{Cytogenetic and FISH analyses}

Routine cytogenetics was performed according to standard GTG-banding protocol, either concurrently with CMA or for confirmation of mosaicism. T cells were stimulated with PHA from peripheral blood collected in sodium heparin tubes and cultured for $72 \mathrm{~h}$. The number of metaphases analyzed ranged from confirmation using only 5 cells, to standard 20 cells, up to 100 cells.

Confirmatory FISH analyses on cultured cells were performed with BACs or fosmid clones using standard procedures.

\section{Chromosomal Microarray Analysis}

BCM V8 OLIGO is a custom-designed array with approximately 180000 interrogating oligonucleotides, manufactured by Agilent Technologies, Inc. (Santa Clara, CA, USA). This array contains the 'best-performing' oligonucleotides (oligos) selected from Agilent's online library (eArray; https:// earray.chem.agilent.com/earray/) and has been further empirically optimized. Genomic features of the V8 OLIGO design include interrogation of all known microdeletion and microduplication syndrome regions as well as pericentromeric and subtelomeric regions and computationally predicted NAHRmediated genomic instability regions flanked by low-copy repeats (LCRs) as previously described. ${ }^{14}$ Also included is exonic coverage for $\sim 1700$ selected known or candidate disease genes as well as introns greater than $10 \mathrm{~kb}$. The entire nuclear genome is covered with an average resolution of $30 \mathrm{~kb}$, excluding LCRs and other repetitive sequences. ${ }^{8}$

\section{Calculation of mosaicism}

All data analysis following feature extraction of the TIFF images were performed using in-house software written in the $\mathrm{R}$ open source statistical software. ${ }^{15}$ Briefly, the normalized red (test) and green (control) intensities extracted from TIFF images are used for the calculation of binary logarithmic ratios (log2-ratio) at each probe. DNAcopy was used to identify regions of copy number loss or gain. ${ }^{16}$ Default settings in DNAcopy were used for segmentation, but with post processing to ensure at least three consecutive same sign probes with strong mean $\log 2$-ratios.

Mosaicism for CNV was modeled as a mixture of two cell populations; one cell population with a normal chromosome complement (either 46,XX or $46, \mathrm{XY}$ ), and the other with a single loss or gain at any particular locus (either segmental or whole chromosome). It is not possible to determine mosaic percentages for cases with multiple cell lines affecting the same overlapping region (see Table 1). We calculated the percentage of mosaicism using the formula

$$
\alpha=(|\delta-1| / 0.5) \times 100
$$

where $\delta=2^{\log \mathrm{R}}$ and $\log \mathrm{R}=$ mean $[\log 2($ Test/Control $)]$.

\section{RESULTS}

Of the 10362 patients evaluated by the V8 OLIGO array, a total of 57 cases involving somatic mosaicism were identified (Table 1, Supplementary Table 1) and classified into the following categories: (1) aneuploidy, including sex chromosome abnormalities and isochromosomes (22 cases), (2) ring or marker chromosomes (12 cases), (3) single deletion/duplication CNVs (11 cases), (4) multiple deletion/duplication CNVs (5 cases), (5) exonic CNVs ( 4 cases), and (6) unbalanced translocations (3 cases). Each case was confirmed by either FISH, chromosome analysis, or both. The range of the level of mosaicism varied depending on the method of analysis: we calculated a range of 5-80\% using CMA data; we observed a range from 10 to $93 \%$ by FISH analysis on cultured cells; and a range from 3 to $96 \%$ by G-banded chromosome analysis. In general, the percentage of mosaicism estimated from CMA is highly correlated when compared to that of FISH analysis (Spearman correlation 0.7255, $P=8.4 \times 10^{-6}$ or Pearson correlation $0.726, P=8.15 \times 10^{-6}, n=29$ ) and chromosome analysis (Spearman correlation $0.791, P=.006$ or Pearson correlation $0.832, P=.0015, n=11)$. Overall, 37\% (21/57) of cases had two or more abnormal cell lines (ranging from two to five abnormal cell lines). Of the eight cases where parental studies were performed, all were found to be de novo.

There were 14 mosaicism cases studied concurrently by CMA and chromosome analysis. Ten cases were concordant (71\%) and four cases had normal chromosome results (29\%). Two of these four discordant cases (cases 4 and 8 ) were mosaic for aneuploidy while the remaining two cases (cases 47 and 54) involved CNVs beyond the resolution of standard chromosome analysis. Overall, mosaic CNVs not detectable by chromosome analysis $(<3 \mathrm{Mb})$ were present in $18 \%$ (10/57) of all mosaic cases.

\section{Aneuploidy}

Aneuploidy including sex chromosomes and isochromosomes represents the most common type of mosaicism observed in 22 cases (39\%) as shown in Table 1/Supplementary Table 1 . The most frequent aneuploidy detected involved a sex chromosome (12 cases). Trisomy 8 was the second most common aneuploidy detected in four cases (cases 1-4, Supplementary Figure 1), followed by trisomy 9 in three cases (cases 5-7). The remaining mosaic aneuploidy cases include two cases of trisomy 14 (cases 8 and 9) and one case of trisomy 21 (case 10). 
Table 1 Mosaic abnormalities detected by CMA

\begin{tabular}{|c|c|c|c|c|c|c|c|c|}
\hline \multirow[b]{3}{*}{ Type } & \multicolumn{8}{|c|}{ Level of mosaicism (\%) } \\
\hline & \multicolumn{3}{|l|}{ Case } & \multirow[b]{2}{*}{ FISH (cell count) } & \multicolumn{2}{|l|}{ Chromosome analysis } & \multicolumn{2}{|l|}{ Number of } \\
\hline & number & Mosaicism & CMA & & (cell count) & Size $(M b)$ & probes & Indication \\
\hline \multirow[t]{22}{*}{ Aneuploidy } & 1 & Trisomy 8 & 80 & $73.5(147 / 200)$ & NA & WC8 & 6993 & No indication provided \\
\hline & 2 & Trisomy 8 & 13 & $10(19 / 200)$ & NA & WC8 & 6993 & DF \\
\hline & 3 & Trisomy 8 & 9 & NA & $23(7 / 30)$ & WC8 & 6993 & $\begin{array}{l}\text { FHx of chromosome } \\
\text { abnormality }\end{array}$ \\
\hline & 4 & Trisomy 8 & 5 & $16(36 / 219)$ & Normal (20) & WC8 & 6993 & $\begin{array}{l}\text { Agenesis of the } \\
\text { corpus callosum }\end{array}$ \\
\hline & 5 & Trisomy 9 & 19 & $11(22 / 195)$ & $14(7 / 50)$ & WC9 & 6944 & $\mathrm{DD}$ \\
\hline & 6 & Trisomy 9 & 51 & $25(51 / 200)$ & NA & WC9 & 7169 & $\begin{array}{l}\text { DF, Hypomelanosis of Ito, } \\
\text { cerebellar hypoplasia }\end{array}$ \\
\hline & 7 & Trisomy 9 & 23 & $\begin{array}{l}20(\times 3)(40 / 200) \\
9(\times 1)(17 / 200)\end{array}$ & NA & WC9 & 7169 & $\begin{array}{l}\text { Mild DD, DF, possible } \\
\text { Rubenstein-Taybi }\end{array}$ \\
\hline & 8 & Trisomy 14 & 16 & $13(26 / 200)$ & Normal (30) & WC14 & 4294 & Speech delay \\
\hline & 9 & Trisomy 14 & 15 & $11(24 / 210)$ & $3(2 / 61)$ & WC14 & 4294 & Hypothyroidism \\
\hline & 10 & Trisomy 21 & 27 & $39(78 / 200)$ & NA & WC21 & 2148 & $\begin{array}{l}\text { Mosaic trisomy } 21 \text {, retinal } \\
\text { dystrophy, SZ, decreased } \\
\text { leg strength }\end{array}$ \\
\hline & 11 & $45, X / 46, x X$ & 30 & NA & $43(13 / 30)$ & wCX & 13252 & Turner syndrome \\
\hline & 12 & $45, X / 46, X X$ & 33 & $55(138 / 250)$ & NA & WCX & 13252 & Extreme FTT, MCA \\
\hline & 13 & $45, X / 46, x X$ & 44 & NA & $56(56 / 100)$ & wCX & 11452 & FTT \\
\hline & 14 & $45, X / 46, x X$ & 29 & NA & $24(6 / 25)$ & wCX & 11450 & $\mathrm{DD}$ \\
\hline & 15 & $45, X / 46, X X$ & 13 & $13(26 / 200)$ & $10(2 / 20)$ & WCX & 11452 & $\begin{array}{l}\text { DF, moderate DD, } \\
\text { microcephaly, } \\
\text { multicystic kidney }\end{array}$ \\
\hline & 16 & $45, X / 46, X X / 47, X X X$ & a & $\begin{array}{l}83(47, X X X)(249 / 300) \\
8(46, X X)(25 / 300) \\
9(45, X)(26 / 300)\end{array}$ & NA & WCX & 11452 & $\begin{array}{l}\text { Moderate DD, mosaic } \\
45, X / 47, X X X\end{array}$ \\
\hline & 17 & $45, X / 46, X Y$ & a & $\begin{array}{l}75(45, X)(112 / 150) \\
23(46, X Y)(34 / 150)\end{array}$ & NA & WCX & 13255 & $\begin{array}{l}\text { Turner syndrome with } \mathrm{XY} \\
\text { cell line }\end{array}$ \\
\hline & 18 & 45,X/46,X,idic(Y) & a & $\begin{array}{l}47(45, X)(47 / 100) \\
53(46, X, \operatorname{idic}(Y))(53 / 100)\end{array}$ & 46,X,idic(Y) (5/5) & $\begin{array}{l}\text { WCX } \\
37.4\end{array}$ & 13228 & $\begin{array}{l}\text { MCA, renal agenesis, DF, } \\
\text { GER }\end{array}$ \\
\hline & 19 & $45, X / 46, X, \operatorname{idic}(Y)$ & a & $\begin{array}{l}57(45, X)(120 / 210) \\
43(46, X, \operatorname{idic}(Y))(90 / 210)\end{array}$ & $\begin{array}{l}40(45, X)(8 / 20) \\
60(46, X, \operatorname{idic}(Y)) \\
(12 / 20)\end{array}$ & $\begin{array}{l}\text { WCX } \\
37.4\end{array}$ & 13229 & $\begin{array}{l}\text { Turner syndrome with } \\
\text { marker }\end{array}$ \\
\hline & 20 & 45,X/46,X,idic(X)(p11.2) & a & $\begin{array}{l}92(45, X)(74 / 80) \text { met } \\
8(46, X, \operatorname{idic}(X)(p 11.22)) \\
(6 / 80) \text { met }\end{array}$ & $100(45, X)(5 / 5)$ & $\begin{aligned} & \text { WCX } \\
\sim & 202.6\end{aligned}$ & 11452 & No indication provided \\
\hline & 21 & $46, X Y / 47, X X Y$ & 36 & $24(47, X X Y)(67 / 275)$ & NA & WCX & 11450 & Microcephaly \\
\hline & 22 & $47, X X Y / 48, X X Y Y$ & a & NA & $\begin{array}{l}96(47, X X Y)(48 / 50) \\
4(48, X X Y Y)(2 / 50)\end{array}$ & WCX WCY & 11450 & $\begin{array}{l}\text { Mild } D D \text {, minor } D F \text {, } \\
\text { radioulnar synostosis, ear } \\
\text { pit }\end{array}$ \\
\hline \multirow[t]{12}{*}{ Ring/Marker } & 23 & $45, X / 46, X, r(X)$ & a & $\begin{array}{l}15(45, X)(3 / 20) \text { met } \\
85(46, X, r(X))(17 / 20) \text { met }\end{array}$ & NA & $\begin{array}{l}\text { WCX } \\
9.755\end{array}$ & 13252 & No indication provided \\
\hline & 24 & $45, X / 46, X, r(X)$ & a & $\begin{array}{l}85(45, X)(170 / 200) \\
15(46, X, r(X))(30 / 200)\end{array}$ & NA & $\begin{array}{l}\text { WCX } \\
6.691\end{array}$ & 13255 & $\begin{array}{l}\text { Abnormal chromosome } \\
\text { analysis, severe DD, SZ }\end{array}$ \\
\hline & 25 & $45, X / 46, X, r(X)$ & a & $\begin{array}{l}49(45, X)(98 / 200) \\
51(46, X, r(X)(102 / 200)\end{array}$ & $\begin{array}{l}33(45, X)(5 / 15) \\
67(46, X, r(X))(10 / 15)\end{array}$ & $\begin{array}{l}\text { WCX } \\
7.31\end{array}$ & 13252 & FTT \\
\hline & 26 & $45, X / 46, X, r(X)$ & a & $\begin{array}{l}71(45, X)(142 / 200) \\
29(46, X, r(X))(58 / 200)\end{array}$ & NA & $\begin{array}{l}\text { WCX } \\
45.5\end{array}$ & 4870 & $\begin{array}{l}\text { Turner syndrome, ring } \\
\text { chromosome }\end{array}$ \\
\hline & 27 & $45, X / 46, X, r(X)$ & a & $\begin{array}{l}46(45, X)(118 / 254) \\
54(46, X, r(X))(136 / 254)\end{array}$ & $\begin{array}{l}55(45, X)(11 / 20) \\
45(46, X, r(X))(9 / 20)\end{array}$ & $\begin{array}{l}\text { WCX } \\
25.88\end{array}$ & 11450 & DF, Short stature \\
\hline & 28 & $45, X / 46, X, r(X)$ & a & NA & $\begin{array}{l}50(45, X)(5 / 10) \\
50(46, X, r(X))(5 / 10)\end{array}$ & $\begin{array}{l}\text { WCX } \\
52.99\end{array}$ & 11452 & Moderate DD, DF \\
\hline & 29 & $r(2)(q 11.1 q 12.1)$ & 27 & $46(92 / 200)$ & $\begin{array}{l}50(47, X X,+ \text { mar }) \\
(10 / 20)\end{array}$ & 7.2 & 327 & Chromosome abnormality \\
\hline & 30 & $r(6)(p 12 q 12)$ & a & $\begin{array}{l}57 \text { (one ring)(114/200) } \\
9.5 \text { (two rings) }(19 / 200) 33.5 \\
\text { (no rings) }(67 / 200)\end{array}$ & $\begin{array}{l}61(47, X X,+r) \\
(61 / 100) 30 \\
(48, X X,+r 1,+r 2) \\
(30 / 100) 9(46, X X) \\
(9 / 100)\end{array}$ & $\begin{array}{l}\text { r1 } 8.56 \\
\text { r2 } 15.08\end{array}$ & 599 & $\begin{array}{l}\text { Moderate DD, DF, ring } \\
\text { chromosome }\end{array}$ \\
\hline & 31 & $r(8)(p 11.21 q 12.1)$ & 43 & $43(180 / 420)$ & $\begin{array}{l}55(47, X Y,+r) \\
(11 / 20)\end{array}$ & 15.0 & 542 & DF, hypotonia \\
\hline & 32 & $r(19)(p 13.11 q 13.32)$ & 21 & $13(5 / 40)$ met & NA & 31.3 & 1650 & Moderate DD,DF \\
\hline & 33 & inv dup(15)(q11.2q13.3) & $\mathrm{b}$ & $92(184 / 200)$ & NA & 11.9 & 1316 & Chromosome anomaly \\
\hline & 34 & $\operatorname{mar}(21)$ & 49 & $63(126 / 200)$ & $52(11 / 21)$ & 17.5 & 708 & $\begin{array}{l}\text { DD, MCA, FHx of } 6 p 24.1 \\
\text { dup }\end{array}$ \\
\hline
\end{tabular}


Table 1 (Continued)

Level of mosaicism (\%)

\begin{tabular}{|c|c|c|c|c|c|c|c|c|}
\hline \multirow[b]{2}{*}{ Type } & \multicolumn{2}{|l|}{ Case } & \multirow[b]{2}{*}{ CMA } & \multirow[b]{2}{*}{ FISH (cell count) } & \multirow{2}{*}{$\begin{array}{l}\text { Chromosome analysis } \\
\text { (cell count) }\end{array}$} & \multirow[b]{2}{*}{ Size $(M b)$} & \multirow{2}{*}{$\begin{array}{c}\text { Number of } \\
\text { probes }\end{array}$} & \multirow[b]{2}{*}{ Indication } \\
\hline & number & Mosaicism & & & & & & \\
\hline \multirow{11}{*}{$\begin{array}{l}\text { Single } \\
\text { Dup/Del }\end{array}$} & 35 & $\operatorname{del}(2)(q 24.2 q 24.2)$ & 50 & $61(125 / 204)$ & NA & 0.318 & 42 & DD \\
\hline & 36 & $\operatorname{del}(2)(q 37.3 q 37.3)$ & 48 & $65(137 / 210)$ & NA & 0.664 & 110 & SZ disorder \\
\hline & 37 & $\operatorname{del}(4)(\mathrm{q} 21.21 \mathrm{q} 21.21)$ & 40 & $50(105 / 210)$ & NA & 2.5 & 70 & $\begin{array}{l}\text { Moderate DD, motor delay, } \\
\text { FHx mood disorders }\end{array}$ \\
\hline & 38 & $\operatorname{del}(5)(p 15.3 p 15.3)$ & 41 & $50(101 / 200)$ & NA & 15.5 & 927 & Moderate DD \\
\hline & 39 & $\operatorname{del}(17)(p 13.3 p 13.2)$ & 33 & $40(79 / 200)$ & NA & 1.7 & 196 & Epilepsy \\
\hline & 40 & $\operatorname{del}(18)(q 21.2 q 23)$ & 21 & $22(45 / 200)$ & $13(4 / 30)$ & 28.2 & 1115 & $\begin{array}{l}\text { Congenital vertical talus, } \\
\text { microcephaly }\end{array}$ \\
\hline & 41 & $\operatorname{del}(22)(q 13.2 q 13.3)$ & 34 & $40(89 / 200)$ & NA & 8.4 & 303 & Moderate DD \\
\hline & 42 & $\operatorname{del}(22)(q 13.31 q 13.3)$ & 49 & $58(29 / 50)$ & NA & 6.9 & 271 & Medulloblastoma \\
\hline & 43 & dup(2)(p24.3p22.3) & 49 & $65(137 / 210)$ & NA & 21.1 & 1012 & No indication provided \\
\hline & 44 & $\operatorname{dup}(12)(p 13.33 p 11.1)$ & $\mathrm{b}$ & $\begin{array}{l}50(x 4)(100 / 200) 12.5(x 3) \\
(25 / 200) 37.5(x 2)(75 / 200)\end{array}$ & NA & 33.9 & 2524 & Dysmorphic features \\
\hline & 45 & $\operatorname{dup}(12)(\mathrm{p} 13.3 \mathrm{p} 11.22)$ & 48 & $47(14 / 30) \mathrm{met}^{\mathrm{C}}$ & NA & 28.4 & 2267 & Chromosomal abnormality \\
\hline \multirow[t]{5}{*}{$\begin{array}{l}\text { Multiple } \\
\text { Dup/Del }\end{array}$} & 46 & Trisomy $9 \operatorname{der}(9)$ & a & $\begin{array}{l}61(+9)(183 / 300) 26(\operatorname{der}(9)) \\
(78 / 300)\end{array}$ & NA & 140.0 & 6944 & MCA \\
\hline & 47 & $\operatorname{del}(6)(p 21.33 p 21.32)$ & (6)49 & (6)50 (31/60) (17)50 (101/200) & Normal (20) & 0.338 & (6) 27 & SZ, DD \\
\hline & 48 & $\begin{array}{l}\operatorname{del}(1 /)(p 11.2) \\
\operatorname{del}(17)(p 13.3 p 13.3) \\
\operatorname{del}(17)(p 13.3 p 13.1)\end{array}$ & $\begin{array}{c}(1 /) 34 \\
a\end{array}$ & $\begin{array}{l}77.5 \text { (del 17)(p13.3p13.3) } \\
(31 / 40) 22.5(\text { del } \\
17)(p 13.3 p 13.1)(9 / 40)\end{array}$ & $\begin{array}{l}18(\text { del17p } 13.2) \\
(5 / 30) 82(46, X Y) \\
(25 / 30)\end{array}$ & $\begin{array}{l}0.918 \\
1.85 \\
6.8\end{array}$ & $\begin{array}{c}(1 /) 52 \\
211 \\
582\end{array}$ & Epilepsy \\
\hline & $49^{d}$ & $\begin{array}{l}\operatorname{dup}(22)(q 13.31 q 13.32) \\
\operatorname{del}(22)(q 13.32 q 13.33)\end{array}$ & a & $\begin{array}{l}6(x 5)(17 / 300) 36(x 4)(109 / 300) \\
17(x 3)(50 / 300) 10(x 2)(29 / 300) \\
31(x 1)(95 / 300)\end{array}$ & NA & $\begin{array}{l}1.852 \\
4.932\end{array}$ & & Moderate DD \\
\hline & 50 & $\begin{array}{l}\text { 45,X/der(X) } \\
\operatorname{dup}(X)(q 21.32 q 28) \\
\operatorname{del}(X)(q 28 q 28)\end{array}$ & a & $\begin{array}{l}40(\operatorname{der}(X))(4 / 10) \text { met } 60(45, X) \\
(6 / 10) \text { met } 36(\operatorname{del}(X))(19 / 53) \text { int }\end{array}$ & NA & 154.6 & 11452 & No indication provided \\
\hline \multirow[t]{4}{*}{ Single Gene } & 51 & $\begin{array}{l}\operatorname{del}(6)(q 23.1 q 23.2) \\
(E P B 41 L 2)\end{array}$ & 46 & $46(67 / 145)$ & NA & 0.119 & 62 & Autistic spectrum \\
\hline & 52 & del(18)(q21.2) (TCF4) & 61 & $16(32 / 200)$ & NA & 0.185 & 38 & $\begin{array}{l}\text { DF, ADHD, anxiety, self- } \\
\text { mutilation, stiff joints }\end{array}$ \\
\hline & 53 & $\operatorname{del}(\mathrm{X})(\mathrm{p} 11.4)(\mathrm{CASK})$ & 52 & $58(122 / 210)$ & NA & 0.134 & 32 & Moderate DD, FTT, DF \\
\hline & 54 & $\operatorname{del}(\mathrm{X})(\mathrm{p} 21.12)($ IL1RAPL1) & 51 & $56(112 / 200)$ & Normal (20) & 0.035 & 18 & DD, PDD NOS \\
\hline Unbalanced & 55 & $\operatorname{der}(17) t(5 ; 17)(p 14.1 ; p 13.3)$ & $\operatorname{der}(17) 28$ & $40(\operatorname{der} 17)(4 / 10)$ & $25(\operatorname{der} 17)(5 / 20)$ & (5)28.6 & (5) 1558 & DD \\
\hline \multirow[t]{3}{*}{ Translocations } & & $\operatorname{del}(17)(p 13.3)$ & $\operatorname{del}(17) N A$ & 60 (del 17p13.3) (6/10) & (del 17) NA & $(17) 1.9$ & (17)222 & \\
\hline & 56 & $\operatorname{der}(\mathrm{X}) \mathrm{t}(\mathrm{X} ; 1)(\mathrm{p} 21.1 ; \mathrm{q} 21.1)$ & $\operatorname{der}(X) 14$ & $6.5(\operatorname{der}(X))(13 / 200)$ & NA & $\begin{array}{l}(1) 103.4 \\
(X) 34.5\end{array}$ & $\begin{array}{l}(1) 6782 \\
(X) 2872\end{array}$ & No indication provided \\
\hline & 57 & $\begin{array}{l}45, X \\
\operatorname{der}(X) t(X ; 10)(q 25 ; p 11.2)\end{array}$ & a & $\begin{array}{l}6(45, \mathrm{X})(12 / 200) 93(\operatorname{der}(\mathrm{X})) \\
(188 / 200)\end{array}$ & $\begin{array}{l}6(45, X)(3 / 50) 93 \\
(\operatorname{der}(X))(47 / 50)\end{array}$ & $\begin{array}{c}\text { WCX } \\
(10) 34.6\end{array}$ & $\begin{array}{l}(X) 11452 \\
(10) 1595\end{array}$ & DD \\
\hline
\end{tabular}

Abbreviations: ADHD, attention deficit-hyperactivity disorder; CMA, chromosomal microarray analysis; DD, developmental delay; DF, dysmorphic features; FHx, family history; FISH, fluorescence in situ hybridization; FTT, failure to thrive; GER, gastroesophageal reflux; int, interphase; MCA, multiple congenital anomalies; met, metaphase; NA, not available; PDD NOS, pervasive developmental disorder not otherwise specified; SZ, seizures; WC, whole chromosome.

apercent mosaicism estimates by CMA not available due to the presence of more than one abnormal cell line.

bercent mosaicism estimates by CMA not available due to varying multiple copy numbers of the same genomic interval.

'Results obtained from previous cytogenetic testing at another laboratory.

${ }^{d}$ Case 49 was determined to be a ring chromosome 22 following FISH confirmation.

CMA and chromosome results were discordant for cases 4 and 8 . For case 4, the chromosome results from a 20-cell analysis were normal while the CMA plot shows a very slight increase in the intensity ratio for the chromosome 8 region (Supplementary Figure 1, bottom plot), suggesting low-level mosaicism, which was calculated to be $\sim 5 \%$. FISH confirmed the mosaicism for trisomy 8 to be present in $16 \%$ of cells (Supplementary Figures 1a,b,c). Case 8 was mosaic for trisomy 14 (estimated to be $16 \%$ by CMA with FISH confirmation showing $13 \%$ mosaicism) yet was normal by chromosome analysis of 30 cells.

In case 7 , an additional cell line was detected from confirmatory FISH analysis that was not evident from the CMA results. The CMA results suggested mosaic trisomy 9 in $\sim 23 \%$ of cells. FISH confirmation performed on cultured cells detected trisomy 9 in $10 \%$ of cells which was consistent with the CMA estimation, but also revealed a second abnormal cell line with monosomy 9 in $9 \%$ of the cells. Overall, for the aneuploidy cases, the percent of mosaicism estimated by CMA and observed by FISH and/or chromosomes were concordant except for cases 6 and 12, which differed by 26 and 22 percentage points, respectively (Table 1 ).

\section{Ring and marker chromosomes}

Twelve of the mosaicism cases $(21 \%)$ involved either a ring or a marker chromosome. Cases 23-28 involved cells with 45, X and $46, \mathrm{X}, \mathrm{r}(\mathrm{X})$. The ring $\mathrm{X}$ chromosome in these six cases varied in size from a small marker of $\sim 1 \mathrm{Mb}$ in size to a large ring of $\sim 29 \mathrm{Mb}$ (Table 1, Supplementary Table 1). CMA analysis of case 30 suggested a mosaic gain in copy number surrounding the centromeric region in 
chromosome $6(15 \mathrm{Mb})$, suggestive of a mosaic ring for chromosome 6 (Figure 1a1). FISH studies not only confirmed this finding, but also showed three cell lines (Figure 1a2): 57\% had one ring, 10\% had
2 rings, and 33\% contained no ring chromosomes. Each of the four cases with CMA percent mosaicism estimates was concordant with FISH results.

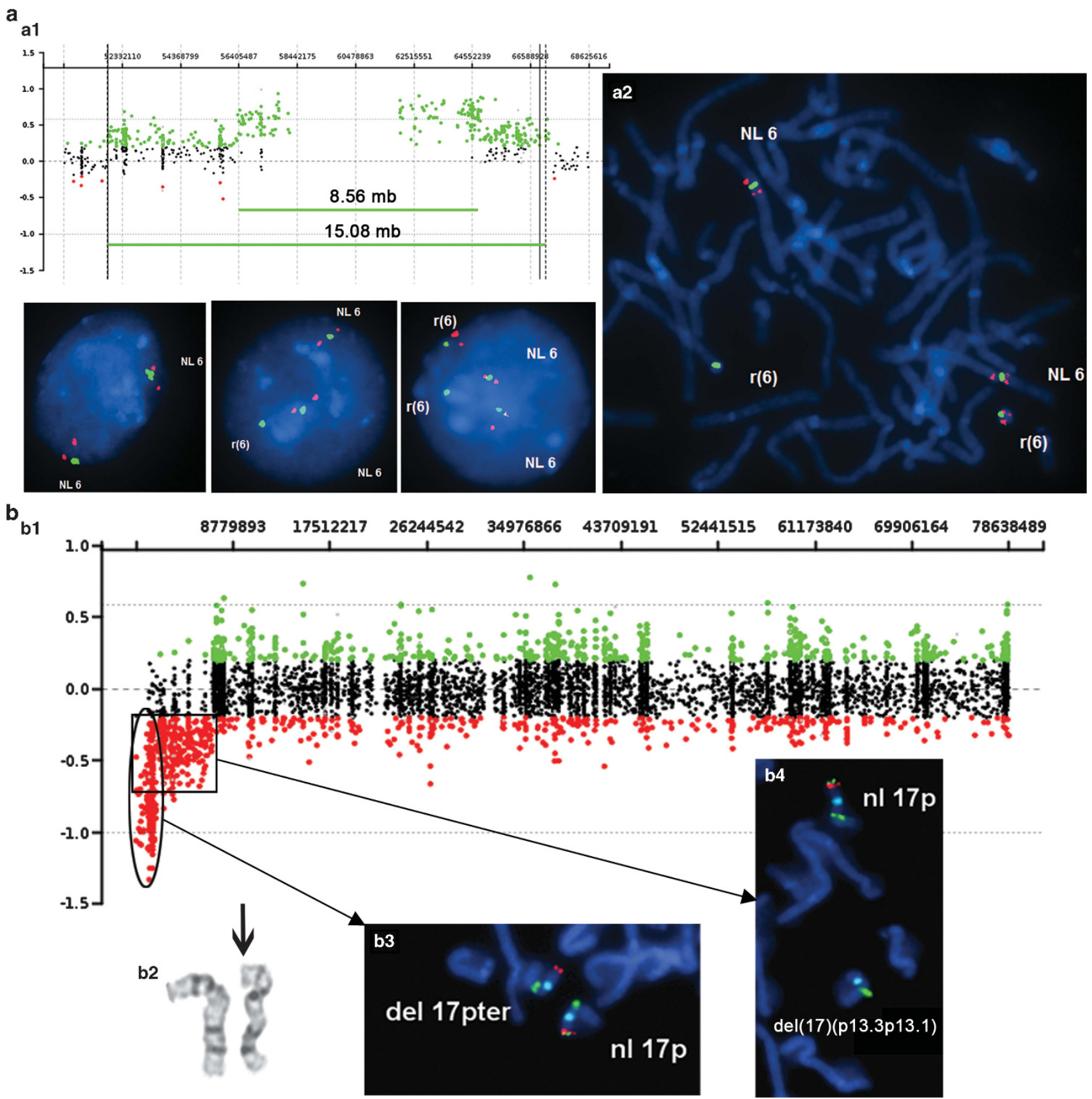

Figure 1 Two illustrative cases with multiple abnormal cell lines. (a) Case 30 is a 20-year-old female with indications of moderate developmental delay, dysmorphic features and a known ring chromosome. (a1) The array plot for the pericentromeric region of chromosome 6 suggestive of two mosaic rings of different sizes as indicated by the green bars. (a2) Metaphase FISH analysis using probes for centromere 6 (green), 6q12 (RP11-586B7 in red), and 6p12.1 (RP11-1055015 in red) confirmed the presence of two rings, each of different sizes. Interphase FISH shows the presence of multiple cell lines containing none, one or two ring 6 chromosomes. (b) Case 48 is a 10-year-old male with epilepsy. (b1) CMA shows two mosaic deletions of different sizes in the short arm of chromosome 17 (circle and square). (b2) Partial karyotype of chromosome 17 from case 48 showing a deletion in the short arm of chromosome 17 indicated by the arrow. Chromosome analysis indicated this deletion is present in 5 out of 30 metaphases (18\%) analyzed. (b3 and b4) FISH analysis using multiple probes - subtelomere $17 \mathrm{p}$ probes cohybridized together (overlapping red and green), LIS1 (red), 17 centromere (aqua), and 17q21.1 control (green) - confirmed cell lines with two different deletions. The circled area on the plot shows a smaller deletion (17p13.3p13.3) present in all cells corresponding to $\mathbf{b 3}$. The deleted 17 on the left of the figure shows the 2 control probes as well as a red signal indicating that the LIS1 gene is still intact. The boxed area in the array plot shows a larger deletion (17p13.3p13.1) corresponding to b4. The chromosome 17 at the bottom of Figure $1 \mathbf{b} 4$ shows a deletion of the LIS1 gene as well as the subtelomere, with signals from only the 17q21 control and centromere probes. 


\section{Single deletions and duplications}

There were 11 cases (cases 35-45) involving a simple segmental abnormality, that is, a single genomic CNV (Table 1, Supplementary Table 1). The size of the CNV ranged from $318 \mathrm{~kb}$ to whole-arm duplications. Eight of the eleven detected mosaic cases were deletions while the remaining three were duplications. Case 35 involves a $318 \mathrm{~kb}$ deletion encompassing the TBR1 gene in 61\% (125/204) of cells. Further details of this case were reported by Burrage et al. ${ }^{17}$ For all cases in which a CMA estimate could be determined, results were concordant.

\section{Multiple deletions and duplications}

Five cases (cases 46-50, Table 1, Supplementary Table 1) involved multiple CNVs which required a combination of methodologies to fully characterize the abnormality. For example, Figure $1 \mathrm{~b}$ shows case 48 , with a complex de novo deletion of the $\mathrm{p}$ arm of chromosome 17 . CMA results showed a complex loss in the $17 \mathrm{p}$ region, suggestive of mosaicism for two different cell lines (Figure 1b1). The concurrent chromosome analysis showed a deletion of $17 \mathrm{p} 13.1$ present in $18 \%$ (5/30) of cells (Figure 1b2). However, FISH studies revealed two abnormal cell lines. The first was present in all cells and consisted of a smaller deletion $(1.832 \mathrm{Mb})$ in the p13.3 region (Figure $1 \mathrm{~b} 3)$. The second was present in 22\% (9/40) of cells and consisted of a larger deletion $(6.784 \mathrm{Mb})$ on the same homologue in the p13.3p13.1 region (Figure 1b4). Case 49 (Supplementary Figure 2) involves both duplication in the 22q13.31q13.32 region (from 46.09 to $48.12 \mathrm{Mb}$ map position hg 18) as well as a deletion in the adjacent $22 \mathrm{q} 13.32 \mathrm{q} 13.33$ region (from 48.16 to $49.52 \mathrm{Mb}$ ). CMA results suggested a complex gain in the proximal q-arm region with a loss in the distal q-arm region (Supplementary Figures 2a,b). Interphase FISH showed five different cell lines for the 22q13.31 region (Supplementary Figure 2c) with one (31\%), two (10\%), three $(17 \%)$, four $(36 \%)$, and five $(6 \%)$ red target signals, respectively, as compared with the control (22q11.21) with two green signals. Upon further analysis, a ring chromosome structure was observed by metaphase FISH, confirming the duplication of the 22q13.31q13.32 segment (Supplementary Figure 2d).

\section{Single genes}

Four cases (cases 51-54, Table 1, Supplementary Table 1) in which copy number losses encompassed or disrupted a single gene were identified. The average number of probes covering the small regions in these four cases was 38 probes, with an average size CNV of $\sim 118 \mathrm{~kb}$. Case 52 involves a $185 \mathrm{~kb}$ deletion of part of the TCF4 gene (Figure 2a-c). Mutations in this gene are associated with Pitt-Hopkins syndrome. ${ }^{18}$ Figure 2 a shows the results of CMA, which suggest a mosaic loss in the long arm of chromosome 18 estimated at $61 \%$. FISH analysis on cultured cells detected the mosaic $18 \mathrm{q} 21.2$ deletion in $16 \%$ of the cells (Figure 2b). The remaining three cases had percent mosaicism estimates by CMA concordant with FISH analysis.

\section{Unbalanced translocations}

Three cases (cases 55-57, Table 1, Supplementary Table 1) involving unbalanced translocations were detected by CMA, including two derivative $\mathrm{X}$ chromosomes, and a derivative 17. Concurrent chromosome analysis for case 55 (Figure $2 \mathrm{~d}$ ) revealed a chromosome 17 with additional material of unknown origin in 25\% (5/20) of cells. CMA results for this case (Figure 2e) suggested an unbalanced translocation between chromosomes 5 and 17. Interestingly, the FISH studies not only confirmed the derivative 17 (Figures $2 \mathrm{f}$ and $\mathrm{g}$ ) but further revealed a second abnormal cell line in which only one copy of 17 p13.3 without the derivative 17 was present (Figure $2 \mathrm{~h}$ ), resulting in monosomy of $17 \mathrm{p} 13.3$ in all cells examined and trisomy of 5 p13.33p14.1 in $25 \%$ of cells. Percent mosaicism estimates by CMA were available only for cases 55 and 56 and are concordant with FISH and chromosome results.

\section{DISCUSSION}

Mosaicism is inherently challenging to detect, because by definition the abnormality will not be present in all cells. Since mosaicism can be confined to specific tissues and not all tissues can be feasibly sampled, it is currently impossible to truly determine the level of mosaicism for a given abnormality in an individual. Despite this seemingly insurmountable difficulty for genomic analyses, technological improvements have enabled the discovery of clinically significant mosaicism not detectable by conventional cytogenetic studies. In this study, we present cases of genomic mosaicism ranging in size from aneuploidy to exonic deletions, the smallest being $35 \mathrm{~kb}$ in size, identified by an exon-targeted high-resolution oligonucleotide array and highlight those cases with discordant results for the different methodologies utilized.

Although the true prevalence of mosaicism in the general population and its incidence are difficult to determine, the detection rate appears to be increasing as more sensitive technologies allow for greater detection. In 1982, Hansteen et al. detected three cases of true mosaicism out of $1830(0.16 \%)$ consecutively born infants by chromosome analysis. ${ }^{19}$ In our study, somatic mosaicism was detected in a total of 57 cases $(0.55 \%)$. Extrapolating from our detection rate, we see a slightly higher incidence of approximately 1 in 180 cases referred for CMA testing. Excluding those cases submitted with an indication of Turner or Down syndrome, which are more likely to have mosaicism, the detection rate was $0.49 \%$. Our detection rate is comparable to previous oligonucleotide and BAC array CGH studies (Supplementary Table 2). In contrast, SNP arrays have been reported to have a higher mosaic detection rate $(1 \%)$ although the rate is more comparable to this study if only comparing blood samples. ${ }^{3}$ While SNP array platforms are considered more sensitive due to the additional data available from the B allele frequency ${ }^{3,4,20}$, a limitation may still exist for detecting single exon CNVs since SNPs predominate in non-coding regions. ${ }^{13}$

The various cytogenetic techniques utilized for detection of mosaicism each have their own strengths and weaknesses. While CMA is able to analyze total DNA from all cells in a given sample (mostly from peripheral blood), conventional cytogenetic analysis relies on PHA-stimulated $\mathrm{T}$ cells. The stimulation and culturing of cells introduces a bias and can lead to a missed diagnosis of mosaicism. ${ }^{1,2}$ There are known syndromes with mosaic chromosome abnormalities, such as the isochromosome $12 \mathrm{p}$ seen in Pallister-Killian syndrome (PKS), that are typically not detected by chromosome studies on peripheral blood. ${ }^{21} \mathrm{~A}$ recent study by Bi et al. examined the value of conventional cytogenetics in the context of an ever-increasing use of CMA technology and concluded that, in relation to detection of mosaicism, chromosome studies remain valuable in detecting low-level mosaicism for either abnormal or normal cell lines. ${ }^{5}$ However, detection of this low level of mosaicism by chromosome studies requires a large number of metaphases to be analyzed, which is labor intensive, and may still not detect mosaicism as exemplified by cases 4 and 8 in this study. Although extremely low levels $(<10 \%)$ of mosaicism may not reliably be detected by CMA, it should be noted that case 4 , which showed an estimated $5 \%$ mosaicism for trisomy 8 , was detected by CMA only. Without 

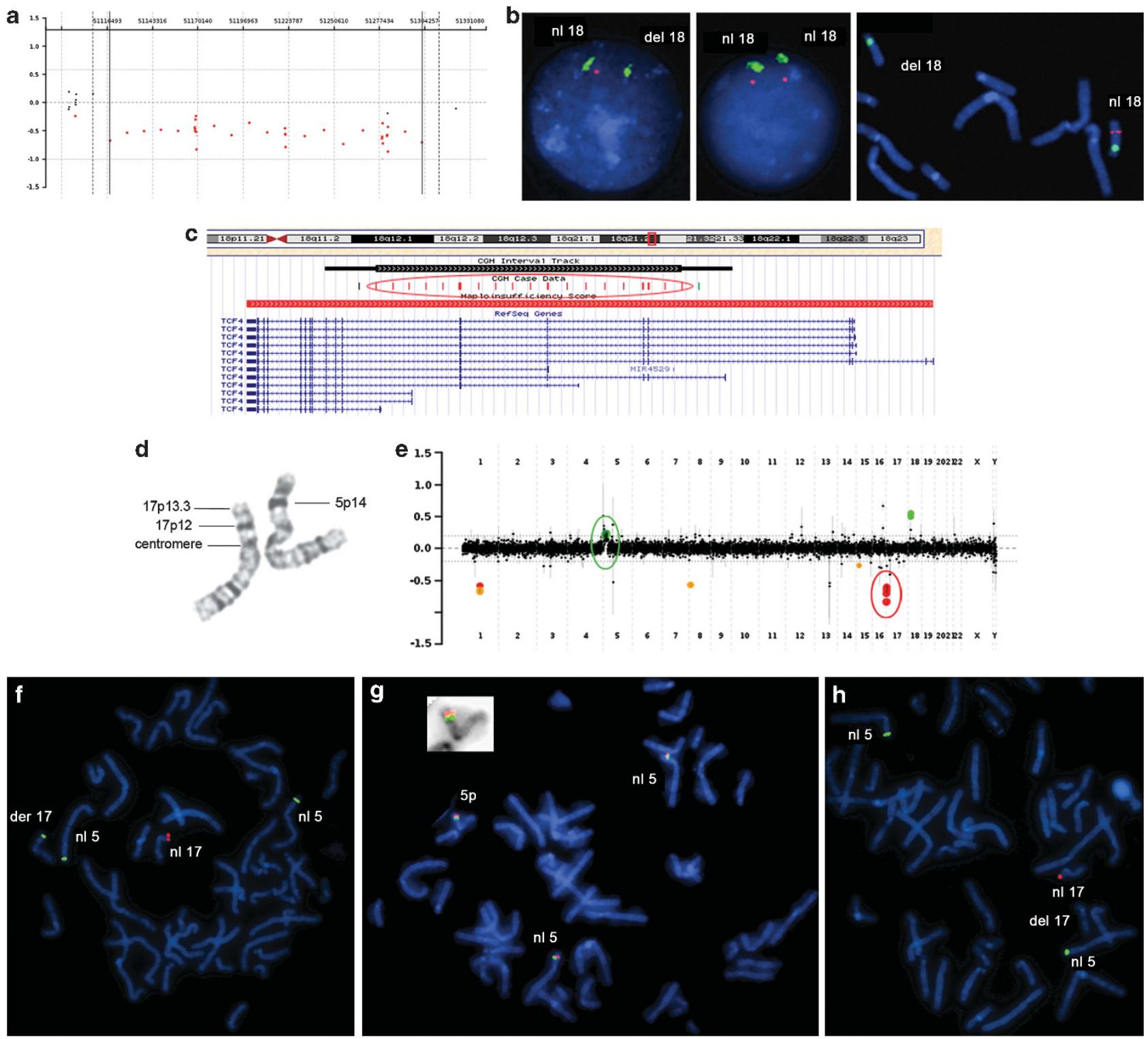

Figure 2 Case 52 is a 17-year-old male with dysmorphic features, attention deficit hyperactivity disorder, anxiety, self-mutilation and stiff joints. (a) An enlarged image of the array plot of chromosome 18q21 showing the region of copy number loss detected by 35 interrogating probes within the TCF4 gene which is associated with Pitt-Hopkins syndrome. (b) FISH on interphase cells confirming the mosaic deletion (probe RP11-111C17 in red at 18q21.2 and centromere 18 in green) present in 16\% of cells (32/200) and a second normal interphase FISH cell. A partial FISH metaphase cell shows the deletion at 18q21.2. (c) Visualization of the deleted exons shown on the UCSC genome browser. (d) Case 55 is a 3-year-old female with developmental delay. Partial karyotype of chromosome 17 showing the additional material on 17p indicated by the arrow. Concurrent chromosome analysis revealed additional material of unknown origin on the short arm of chromosome 17, later revealed to be material from the short arm of chromosome 5. (e) The array plot showing a complete loss of material approximately $1.9 \mathrm{Mb}$ in size in the $17 \mathrm{p} 13.3$ region, circled in red, in addition to a mosaic gain in the short arm of chromosome 5 of $\sim 28.6 \mathrm{Mb}$ in size, circled in green. (f) A FISH probe hybridizing to the $5 \mathrm{p} 15.2$ region (D5S23) is shown in green, while the 17p13.3 region (RP11$411 \mathrm{G} 7)$ is shown in red. Metaphase FISH shows a cell line with a derivative 17 chromosome [der(17)t(5;17)(p14.1;p13.3)], indicated by the green signal on the 17 and absence of a red signal. (g) A FISH probe hybridizing to the 5p14.1 region (RP11-15B5) is shown in green and 5p14.3 (RP11-44L10) in red. Metaphase FISH confirms trisomy for the 5 p13.33p14.1 region seen in $40 \%$ of cells analyzed. The inverted DAPI insert shows a close-up of the derivative 17 chromosome with the material from the 5p. (h) FISH image showing the second cell line. FISH probes for 17p13.3 (RP11-411G7) in red and 5 p15.2 (D5S23) in green showing a deletion in the short arm of chromosome 17 and absence of a derivative 17. Monosomy for the 17p13.3 region was observed in all cells.

dispute, chromosome studies are especially valuable for mosaic cases with multiple cell lines affecting the same overlapping region, rendering the calculation of mosaicism using $\log 2$ intensity values ineffective. FISH provides a clear advantage over chromosome studies in the ability to quickly and easily score hundreds of interphase cells to determine the level of mosaicism, but only when the abnormality has been detected by another assay such as CMA or chromosome analysis, making FISH unsatisfactory as an initial test for detecting unsuspected mosaicism. Both FISH and chromosome analysis aid CMA by providing a visual confirmation, which helps elucidate the complete genome picture and the spectrum of mechanistically observed end products, such as case 48 involving a complex 
deletion. Most interestingly, over a third (21/57) of the cases unexpectedly had multiple abnormal cell lines revealed by FISH and/or chromosome analysis that was not always apparent by CMA. More specifically, over half (13/21) of these cases involved chromosome X. This highlights the importance of performing additional cytogenetic studies for detection of multiple cell lines especially for chromosome $\mathrm{X}$.

Somatic mosaicism detected by CMA but missed by chromosome analysis has been previously reported. ${ }^{1,2,7,22}$ Our array detected two cases of mosaicism, both whole chromosome aneuploidies, that should have been detected by conventional cytogenetic analyses $(>3 \mathrm{Mb})$ yet were normal. Case 4 , mosaic for trisomy 8 , was normal by chromosome analysis of 20 cells and case 8 was mosaic for trisomy 14 yet was normal by chromosome analysis of 30 cells. $^{23}$ Cheung et al. reported 9 out of 12 mosaic cases first detected by CMA with previously normal routine chromosome analysis and 8 of the 9 cases involved whole chromosome aneuploidy. ${ }^{1}$ Ballif et al. reported 6 out of 18 mosaic cases with a normal karyotype. Three of the six were aneuploidy, while two involved deletions $>5 \mathrm{Mb}$ in size, and one involved a mosaic derivative chromosome. ${ }^{2}$ Hoang et al. reported four cases out of twelve for mosaicism with previously normal karyotypes, where three of the four were aneuploidy. ${ }^{22}$ Overall, there appears to be susceptibility for mosaic aneuploidies to be missed by conventional cytogenetics, suggesting a selection process occurring in cultured cells in favor of normal cells. Similar to a mosaic isochromosome 12p, the cytogenetic hallmark of PKS, CMA has been shown to be able to detect low levels of mosaicism of the $\mathrm{i}(12 \mathrm{p})$ in peripheral blood of PKS individuals while the abnormality is not always detected by FISH or chromosome analysis. $^{24}$

One of the main advantages of CMA over chromosome analysis is its ability to detect small CNVs, enabling discovery of mosaicism for abnormalities not previously detectable. Ten cases (18\%) involved CNVs $(<3 \mathrm{Mb})$ that would not be expected to be detectable by conventional cytogenetic studies. Due to the extensive exonic coverage of the custom array used in this study, we were able to detect CNVs involving single genes in four cases, three of which are known to be disease-causing. Case 52 involves a $185 \mathrm{~kb}$ (38 probes) deletion encompassing the TCF4 gene in which haploinsufficiency is known to cause Pitt-Hopkins syndrome. ${ }^{18}$ The finding is consistent with the patient's phenotype, which includes dysmorphic features, ADHD, anxiety, self-mutilation, and stiff joints. ${ }^{8}$ Only two other cases have ever been reported to involve a somatic mosaic deletion of TCF4. ${ }^{25,26}$ Case 53 involves a $134 \mathrm{~kb}$ ( 32 probes) de novo deletion encompassing exons $2-5$ of the CASK gene. Deletions involving this gene have been reported in patients with intellectual disability and microcephaly with pontine and cerebellar hypoplasia (OMIM\# 300749) and Ohtahara syndrome. ${ }^{19}$ The phenotype of this five-year-old male patient (developmental delay, microcephaly, craniofacial dysmorphic features, and speech delay) appears consistent with this diagnosis. Case 54 involved a $35 \mathrm{~kb}$ (18 probes) deletion in the IL1RAPL1 gene which is associated with $\mathrm{X}$-linked intellectual disability (OMIM\# 300206), and also consistent with the patient's phenotype. ${ }^{8}$ Each of these cases involved deletions far beyond the resolution of conventional cytogenetics and would have been missed without CMA testing. Exonic coverage allows for detection of mosaicism at an unprecedented level and highlights the potential for mosaicism involving exons of genes, as a group of abnormalities, to be an underrecognized cause of phenotypic abnormality.

Importantly, there were $35 \%$ (20 of 57 ) of cases where we were unable to estimate the mosaicism levels. These cases typically involved multiple abnormal cell lines and presented the most challenge for interpretation by CMA alone. One abnormality category especially affected is the ring/marker group. Because of the unstable nature of ring/marker chromosomes, they are most frequently seen in the mosaic state and it is not unusual to observe multiple cell lines, such as in case 30 which had cell lines containing none, one or two ring 6 chromosomes. Most interestingly, case 49 showed an astonishing five different cell lines. While FISH analysis showed a ring chromosome structure for this case, this type of chromosome abnormality could also be the result of an inverted duplication and terminal deletion as reported by others. ${ }^{27-29}$ The most common formation of ring chromosomes involves breakage in both arms of a chromosome, resulting in a loss of the distal fragments, followed by fusion of the proximal broken ends as exemplified in cases $23-31 .^{30}$ In contrast, some ring chromosome formations are complex rearrangements leading to concurrent deletion and contiguous duplication such as those seen in case 49 . While the ring itself appears to have remained stable as indicated by the consistent normal copy number for the control probe, the distal duplicated region at band $22 \mathrm{q} 13$ is unstable as evidenced by multiple cell lines ranging in CNVs from one to five suggesting a post-zygotic event involving a multiple-step process. The initial event was likely a terminal deletion $[\operatorname{del}(22)(\mathrm{q} 13.32)]$ that was rescued by a classical ring formation in $31 \%$ of cells (Supplementary Figure $2 \mathrm{C}$ ). In the other $59 \%$ of cells, telomere healing via a breakagefusion-bridge cycle may have occurred, leading to an unstable dicentric chromosome. Its subsequent uneven break likely resulted in a formation of an inverted $\operatorname{dup}(22)(\mathrm{q} 13.31 \mathrm{q} 13.32)$. The head-tohead structure is further supported by the inverted duplication pattern of FISH signals (Supplementary Figure 2E). In 17\% of cells, this chromosome was rescued by a ring formation similar to the ring formation of other acrocentric chromosomes. ${ }^{28}$ In the remaining cells, the unstable inv dup/del chromosome may have been rescued independently two times by aberrant DNA replication such as 'fork stalling and template switching' (FoSTeS) ${ }^{31}$ or a microhomologymediated break-induced replication, ${ }^{32}$ resulting in the q13.31q13.32 fragment being triplicated (36\%) and quadruplicated (6\%) (Supplementary Figure 2D) and forming different ring chromosomes. Replication errors have been recently proposed to explain similar terminal triplication and complex dup-trp/inv-dup structures. ${ }^{33}$ However, the limited resolution of CMA did not reveal such complex structures in our case. Interestingly, the fact that we did not observe any double rings in chromosome analysis and the unchanged log ratio of the 22pter-q13.32 segment in the CMA data are consistent with the mechanism for formation of inv dup del type of rearrangments.

In addition, when the ring or marker chromosome is present in low levels or exists in more than one cell line, its presence can be masked by the major cell line. For example, in case 24, the whole-genome array plot depicts a copy number loss of the entire $\mathrm{X}$ chromosome plus a suspicion of a ring $\mathrm{X}$ chromosome (Supplementary Figure $3 \mathrm{~A}$ ). However, the array plot for the $\mathrm{X}$ chromosome only did not exhibit a pattern suggesting a ring X chromosome (Supplementary Figure 3B). The presence of the ring chromosome was clearly evident from chromosome analysis which showed it to be in $15 \%$ of the cells (Supplementary Figure 3C) and also provided an estimate of the size of the ring which was not discernible by CMA. Similarly, in case 20, the array profile depicted a copy number loss of what appears to be the entire X chromosome (Supplementary Figure 4A,B) while FISH analysis revealed the presence of an isodicentric $\mathrm{X}$ chromosome from Xp11.2 to Xqter in $8 \%$ of the cells with the remaining cells showing 45,X (Supplementary Figure 4C,D). 
Not unsurprisingly, the category with the fewest number of detected mosaic cases were those involving unbalanced translocations, as illustrated by case 53 (Figure $2 \mathrm{~d}-\mathrm{h}$ ). Mosaicism for an unbalanced translocation is rare. Hsu et al. reported $555(0.3 \%)$ cases of chromosomal mosaicism in 179665 prenatal cases and found only 1 unbalanced translocation out of the 57 cases that were mosaic for autosomal structural abnormalities. ${ }^{34}$ Recently, Gijsbers et al. reported three cases with mosaic unbalanced translocations using SNP microarray and reviewed the literature and found a total of 10 cases. ${ }^{35}$ In addition, Robberecht et al. studied nine cases of mosaic structural imbalances, three of which were unbalanced translocations. ${ }^{36}$ Our cases together with those of Gijsbers and Robberecht suggest that array technologies may assist with the detection of more of these types of cases.

The ability to analyze total genomic DNA content in a given sample may provide for a more accurate determination of the level of mosaicism as compared to methods involving selected cell populations such as G-banded chromosome analysis. Overall, our calculation of percent mosaicism by CMA has shown close concordance compared to FISH and/or chromosome results (Table 1). However, three cases showed a variation of greater than 20 percentage points between CMA and FISH: case 6 involving a whole chromosome 9 (51\% CMA vs 25\% FISH), case 12 involving a whole X chromosome (33\% CMA vs 55\% FISH), and case 52, a $185 \mathrm{~kb}$ deletion encompassing the TCF4 gene (61\% CMA vs $16 \%$ FISH). In two of the three cases the estimate of mosaicism was greater for CMA. We speculate this is because CMA analyzes total DNA while FISH was performed on cultured, PHA-stimulated T-cells, perhaps leading to selection against abnormal cells. Recently, Thompson and Compton showed that the majority of lagging chromosomes during cell division do not missegregate but instead segregate accurately to end up in micronuclei in daughter cells. ${ }^{37}$ It is therefore possible that these lagging chromosomes in micronuclei can be recovered during DNA extraction for CMA but lost during the process of preparing interphase nuclei for FISH analysis, such as in case 6. In contrast, it is also possible that certain abnormal cells are selected for, or normal cell lines are selected against, in cultured samples as seen in case 12 .

In summary, our study reinforces the effectiveness of CMA in the detection of somatic mosaicism for a wide variety of genomic abnormalities and demonstrates that the estimated level of mosaicism determined by CMA is comparable to the levels determined by FISH and/or chromosome analysis. Furthermore, our mosaicism detection rate of approximately $0.5 \%$ is in close concordance with that published by others. Importantly, with the addition of exon coverage on the array used for clinical testing, mosaicism can be detected at an unprecedented level of resolution involving exons of single genes opening new opportunities to determine the cause of a subset of phenotypic abnormalities that have been previously under-ascertained. While the methodologies utilized in this study have various strengths and weaknesses as presented, used collectively they each contribute a piece of the complex picture aptly known as mosaicism.

\section{CONFLICT OF INTEREST}

Drs Cheung, Shaw, Patel, Stankiewicz, Bi, Lalani, Bacino, and Lupski are faculty members of the Department of Molecular and Human Genetics at Baylor College of Medicine, which derives revenue from the CMA offered in the Medical Genetics Laboratory. Dr Lupski is a consultant for Athena Diagnostics and Ion Torrent Systems, has stock ownership in 23andMe, and is a co-inventor on multiple United States and European patents for DNA diagnostics. All other co-authors declare no conflict of interest.

\section{ACKNOWLEDGEMENTS}

We express our sincere gratitude to the referral physicians, patients and their families. We would also like to thank the cytogenetics laboratories and the CMA laboratory within the Medical Genetics Laboratories for their technical assistance.

1 Cheung SW, Shaw CA, Scott DA et al: Microarray-based CGH detects chromosomal mosaicism not revealed by conventional cytogenetics. Am J Med Genet A 2007; 143A: 1679-1686.

2 Ballif BC, Rorem EA, Sundin K et al: Detection of low-level mosaicism by array $\mathrm{CGH}$ in routine diagnostic specimens. Am J Med Genet A 2006; 140: 2757-2767.

3 Conlin LK, Thiel BD, Bonnemann CG et al: Mechanisms of mosaicism, chimerism and uniparental disomy identified by single nucleotide polymorphism array analysis. Hum Mol Genet 2010; 19: 1263-1275.

4 Bruno DL, White SM, Ganesamoorthy D et al: Pathogenic aberrations revealed exclusively by single nucleotide polymorphism (SNP) genotyping data in 5000 samples tested by molecular karyotyping. J Med Genet 2011; 48: 831-839.

$5 \mathrm{Bi}$ W. Borgan C, Pursley AN et al: Comparison of chromosome analysis and chromosomal microarray analysis: what is the value of chromosome analysis in today's genomic array era? Genet Med 2012; 15:6 450-457.

6 Hassold TJ, Jacobs PA: Trisomy in man. Ann Rev Genet 1984; 18: 69-97.

7 Lu X, Shaw CA, Patel A et al: Clinical implementation of chromosomal microarray analysis: summary of 2513 postnatal cases. PloS One 2007; 2: e327.

8 Boone PM, Bacino CA, Shaw CA et al: Detection of clinically relevant exonic copy-number changes by array CGH. Hum Mutat 2010; 31: 1326-1342.

9 Wiszniewska J, Bi W, Shaw $\mathrm{C}$ et al: Combined array CGH plus SNP genome analyses in a single assay for optimized clinical testing. Eur J Hum Genet 2014; 22: 79-87.

10 Papenhausen P, Schwartz S, Risheg $\mathrm{H}$ et al: UPD detection using homozygosity profiling with a SNP genotyping microarray. Am J Med Genet A 2011; 155A: 757-768.

11 Castronovo C, Rusconi D, Crippa M et al: A novel mosaic NSD1 intragenic deletion in a patient with an atypical phenotype. Am J Med Genet A 2013; 161: 611-618.

12 Saitsu $\mathrm{H}$, Kato M, Osaka $\mathrm{H}$ et al: CASK aberrations in male patients with Ohtahara syndrome and cerebellar hypoplasia. Epilepsia 2012; 53: 1441-1449.

13 Bartnik M, Derwinska K, Gos M et al: Early-onset seizures due to mosaic exonic deletions of CDKL5 in a male and two females. Genet Med 2011; 13: 447-452.

14 El-Hattab AW, Smolarek TA, Walker ME et al: Redefined genomic architecture in $15 q 24$ directed by patient deletion/duplication breakpoint mapping. Hum Genet 2009; 126: 589-602.

15 Core Team R: R: A language and Environment For Statistical Computing. Vienna, Austria: R Foundation for Sttatistical Computing, 2012.

16 Olshen $A B$, Venkatraman ES, Lucito $R$, Wigler M: Circular binary segmentation for the analysis of array-based DNA copy number data. Biostatistics 2004; 5 : 557-572.

17 Burrage LC, Eble TN, Hixson PM, Roney EK, Cheung SW, Franco LM: A mosaic 2q24.2 deletion narrows the critical region to a $0.4 \mathrm{Mb}$ interval that includes TBR1, TANK, and PSMD14. Am J Med Genet A 2013; 161: 841-844.

18 Ardinger HH, Welsh HI, Saunders CJ: Pitt-Hopkins Syndrome 1993.

19 Hansteen IL, Varslot K, Steen-Johnsen J, Langard S: Cytogenetic screening of a new-born population. Clin Genet 1982; 21: 309-314.

20 Rodriguez-Santiago B, Malats N, Rothman N et al: Mosaic uniparental disomies and aneuploidies as large structural variants of the human genome. Am J Hum Genet 2010; 87: 129-138

21 Hodge JC, Hulshizer RL, Seger P St, Antoine A, Bair J, Kirmani S: Array CGH on unstimulated blood does not detect all cases of Pallister-Killian syndrome: a skin biopsy should remain the diagnostic gold standard. Am J Med Genet A 2012; 158A: 669-673.

22 Hoang S, Ahn J, Mann K et al: Detection of mosaicism for genome imbalance in a cohort of 3,042 clinical cases using an oligonucleotide array CGH platform. Eur J Med Genet 2011; 54: 121-129.

23 Hook EB: Exclusion of chromosomal mosaicism: tables of $90 \%$, 95\% and 99\% confidence limits and comments on use. Am J Hum Genet 1977; 29: 94-97.

24 Theisen A, Rosenfeld JA, Farrell SA et al: aCGH detects partial tetrasomy of $12 \mathrm{p}$ in blood from Pallister-Killian syndrome cases without invasive skin biopsy. Am J Med Genet A 2009; 149A: 914-918.

25 Stavropoulos DJ, MacGregor DL, Yoon G: Mosaic microdeletion 18q21 as a cause of mental retardation. Eur J Med Genet 2010; 53: 396-399.

26 Steinbusch CV, van Roozendaal KE, Tserpelis D et al: Somatic mosaicism in a mother of two children with Pitt-Hopkins syndrome. Clin Genet 2013; 83: 73-77.

27 Knijnenburg J, van Haeringen A, Hansson KB et al: Ring chromosome formation as a novel escape mechanism in patients with inverted duplication and terminal deletion. EJHG 2007; 15: 548-555. 
28 Rossi E, Riegel M, Messa J et al: Duplications in addition to terminal deletions are present in a proportion of ring chromosomes: clues to the mechanisms of formation. J Med Genet 2008; 45: 147-154.

29 Guilherme RS, Meloni VF, Kim CA et al: Mechanisms of ring chromosome formation, ring instability and clinical consequences. BMC Med Genet 2011; 12: 171.

30 Kosztolanyi G: Does "ring syndrome" exist? An analysis of 207 case reports on patients with a ring autosome. Hum Genet 1987; 75: 174-179.

31 Lee JA, Carvalho CM, Lupski JR: A DNA replication mechanism for generating nonrecurrent rearrangements associated with genomic disorders. Cell 2007; 131 1235-1247.

32 Hastings PJ, Ira G, Lupski JR: A microhomology-mediated break-induced replication model for the origin of human copy number variation. PLoS Genet 2009; 5 e1000327.

33 Yatsenko SA, Hixson P, Roney EK et al: Human subtelomeric copy number gains suggest a DNA replication mechanism for formation: beyond breakage-fusion-bridge for telomere stabilization. Hum Genet 2012; 131: 1895-1910.

$34 \mathrm{Hsu}$ LY, Yu MT, Richkind KE et al: Incidence and significance of chromosome mosaicism involving an autosomal structural abnormality diagnosed prenatally through amniocentesis: a collaborative study. Prenatal Diagn 1996; 16: $1-28$.

35 Gijsbers AC, Dauwerse JG, Bosch CA et al: Three new cases with a mosaicism involving a normal cell line and a cryptic unbalanced autosomal reciprocal translocation. Eur J Med Genet 2011; 54: e409-e412.

36 Robberecht C, Voet T, Utine GE et al: Meiotic errors followed by two paralle postzygotic trisomy rescue events are a frequent cause of constitutional segmental mosaicism. Mo Cytogenet 2012; 5: 19.

37 Thompson SL, Compton DA: Chromosome missegregation in human cells arises through specific types of kinetochore-microtubule attachment errors. Proc Natl Acad Sci US A 2011; 108: 17974-17978.

(c) (1) () () This work is licensed under a Creative Commons Attribution-NonCommercial-NoDerivs 3.0 Unported License. To view a copy of this license, visit http://creativecommons. org/licenses/by-nc-nd/3.0/

Supplementary Information accompanies this paper on European Journal of Human Genetics website (http://www.nature.com/ejhg) 